\title{
Propensity Score Analysis of Flow Diverters Placed in Scaffolding Stents
}

\author{
(D) A. Poker, (D) Öcal, (DE. Öztürk, and (D)A. Arat
}

\begin{abstract}
BACKGROUND AND PURPOSE: Flow-diverter deployment within a stent remains controversial, but flow-diverter deployment within a scaffolding stent has been performed occasionally. To date, an analysis of this scaffolding technique has not been reported. We aimed to evaluate whether the scaffolding technique adversely affects the outcomes of flow diversion.
\end{abstract}

MATERIALS AND METHODS: Patients who had undergone intracranial aneurysm treatment using a Silk flow diverter with (scaffolding group) or without (bare flow-diverter group) a scaffolding stent were identified retrospectively and compared. Propensity score matching was used to match the aneurysms in both groups for variables with a significant difference between groups. Aneurysm occlusion rates and clinical outcomes were compared.

RESULTS: There were 84 patients (105 aneurysms) in the bare flow-diverter group and 21 patients (22 aneurysms) in the scaffolding group (using 20 LEO stents and 1 Enterprise stent). The aneurysms in the scaffolding group were larger (mean, 13.1 [SD, 10.7] versus 7 [SD, 4.5] $\mathrm{mm}, P=.001)$ and more likely to be fusiform (40.9\% versus $5.7 \%, P<.001)$ than in the bare flow-diverter group. After $2: 1$ propensity score matching, 24 aneurysms in the bare flow-diverter group and 15 in the scaffolding group were matched. Aneurysm occlusion rates did not significantly differ between groups at $1-3$ months (41.2 versus $33.3 \%, P>.99), 3-6$ months $(55.5$ versus $75.0 \%$, $P=.44)$, $7-12$ months (65.0 versus $90.0 \%, P=.21)$, or beyond 1 year $(73.6$ versus $91.6 \%, P=.36)$. There was no difference in complication rates between the groups $(P>.99)$.

CONCLUSIONS: Placement of a scaffolding stent before flow diversion does not adversely affect aneurysm occlusion or complication rates.

ABBREVIATIONS: $B G$ = bare flow-diverter group; $F D$ = flow diverter; SG = scaffolding group

T he Silk Plus stent (Balt Extrusion) has been used in the treatment of intracranial aneurysms with a favorable safety and efficacy profile. ${ }^{1-3}$ However, the deployment of flow diverters (FDs), including the Silk device, may be associated with technical difficulties. Placement of a flow diverter for the treatment of very large-neck or fusiform aneurysms can result in technical adverse events such as migration or prolapse of the device. ${ }^{4}$ Due to these difficulties, a "scaffolding technique," in which a scaffold with a stent is created during the same procedure and just before the placement of a Silk device, has been

Received October 9, 2020; accepted after revision December 9.

From the Departments of Radiology (A.P., O.Ö., A.A.) and Biostatistics (E.Ö.), Faculty of Medicine, Hacettepe University, Sihhiye 06100, Ankara, Turkey; and Department of Radiology (O.Ö.), University Hospital, Ludwig Maximilian University of Munich, Munich, Germany.

Please address correspondence to A. Arat, MD, Department of Radiology, School of Medicine, Hacettepe University, Ankara, Turkey; e-mail: anilarat@hotmail.com

Indicates article with online supplemental data. http://dx.doi.org/10.3174/ajnr.A7040 reported. ${ }^{1,3,5}$ However, as a rule of thumb, placement of a flow diverter within an indwelling stent has been contraindicated. ${ }^{6}$ Most interesting, this suggestion has neither been validated nor challenged to date because no study has compared the results of placement of bare (classic) flow diverters with those of scaffolded flow diverters. We conducted this study to compare the clinical and angiographic results of aneurysms treated by primary stent placement with the Silk device with those treated by Silk device placement in a freshly deployed scaffolding stent.

\section{MATERIALS AND METHODS Study Population}

This retrospective study was approved by the Ethics Committee of our hospital. All intracranial aneurysms treated by a single operator using a Silk Plus flow diverter between April 2010 and March 2019 were identified. Patients were excluded if an apposing stent was deployed inside the Silk device and if a stent or flow diverter had been deployed in a previous session for the same 
aneurysm. Only those patients who were treated with the Silk device, directly or together with a scaffolding stent placed during the same procedure were included. Patients with concurrent endosaccular treatment were not excluded. In the case of tandem aneurysms treated with a single Silk device, both aneurysms were included in the analysis.

The age and sex of the patients and aneurysm location, maximum size, morphology, and history of rupture were recorded. Procedural details and clinically relevant adverse events, that is, any event resulting in a change in $\mathrm{mRS}$, were recorded. Followup imaging results were evaluated on the basis of the RaymondRoy scale. Aneurysms beyond the dural ring in the anterior circulation and above the superior cerebellar arteries in the posterior circulation were defined as distal circulation aneurysms, and the remaining aneurysms were denoted as proximal. The patients treated with a single flow diverter (bare FD group [BG]) and those treated with placement of a scaffolding stent and then a flow diverter in the same session (scaffolding group [SG]) were matched and compared.

\section{General Description of the Interventional Procedure}

The specifications, technical properties, and procedural details related to the Silk device have been described previously by different authors in the literature. ${ }^{2,6,7}$ Patients were administered $75 \mathrm{mg}$ of clopidogrel and $300 \mathrm{mg}$ of acetylsalicylic acid at least 5 days before the procedure and tested with a point-of-care test (VerifyNow P2Y12 assay; Accumetrics). We proceeded with treatment if the inhibition level, in percentages, was $>40 \%$. When it was available, patients were also tested simultaneously with the Multiplate test (Multiplate Analyzer; Roche; platelet inhibition calculated as 1$\mathrm{ADP} / \mathrm{TRAP}$ (thrombin receptor-activating peptide) ratio $\times 100$ ), and in these cases, concordance of the results was sought. If there was high on-treatment platelet reactivity in either test or both tests, these patients were switched from clopidogrel to $10 \mathrm{mg} /$ day prasugrel. Patients treated in the acute-subacute period of subarachnoid hemorrhage were given a loading dose of $600 \mathrm{mg}$ of clopidogrel.

All procedures were performed with the patient under general anesthesia via a transfemoral route in biplane angiography suites. After we catheterized the parent artery with a $6 \mathrm{~F}$ guiding sheath and a distal-access catheter, the aneurysm was bypassed with a microcatheter $(V a s c o+21$ or 25 [Balt] for the Silk Plus flow diverter and Excelsior SL 10 [Stryker] for the Silk Vista baby device) over a microguidewire, and the flow diverter was navigated through this catheter and deployed. The patients treated by this standard method were grouped as the BG.

If we encountered problems associated with device shortening, device prolapse into the aneurysm, excessive device expansion, or size discrepancy between the diameters of the distalversus-proximal landing zones during the deployment of the Silk device, we navigated the deployment catheter over the partially deployed FD, resheathed the device, and proceeded with catheter navigation until the normal segment of the parent artery was reached distally. We then withdrew the device totally and deployed a stent through the indwelling microcatheter (mainly the LEO stent family [Balt] or an Enterprise self-expanding stent [Codman \& Shurtleff]). The use of the Enterprise stent was preferred only when the parent artery was straight and the distal parent artery was not tortuous. Its use was advantageous while deploying the FD because it did not impede the visualization of the FD. On the other hand, the use of this stent required an additional microcatheter exchange maneuver.

Less commonly, in the case of a fusiform aneurysm with a long segment of a diseased parent artery or in wide-neck aneurysms over arterial curves in which we anticipated a risk of device prolapse into the aneurysm, we directly proceeded with stent deployment without a preceding trial of flow-diverter deployment. The scaffolding stent was deployed across the aneurysm neck with at least a 5-mm landing zone on both ends. When this stent was used, the flow diverter was undersized so that the fully expanded diameter matched the maximum diameter of the deployed stent in situ. We undersized the flow diverter so that at its unconstrained, maximum expansion (beyond the nominal diameter), it would match the nominal size of the stent. In general, the nominal diameter of the flow diverter would be $0.5 \mathrm{~mm}$ lower than that of the stent. When possible, we preferred to deploy the flow diverter so that it would cover the whole length of the scaffolding stent. This deployment was frequently not possible due to the foreshortening of the Silk device. In these cases, we preferred to cover as much of the stent as possible proximally. The group treated with these procedures was noted as the SG.

After the procedure, the patients were imaged at 1-3 months by CT or MR angiography, at 3-6 months by DSA, and then at 9-12 months by noninvasive angiography. Further follow-up was scheduled on a patient-by-patient basis by noninvasive imaging. Patients were kept on dual-antiplatelet therapy for 6 months, and clopidogrel (or prasugrel) was discontinued after the 6-month DSA.

\section{Statistical Analysis}

Continuous variables are reported as mean [SD]. Categoric variables were compared using the $\chi^{2}$ test or Fisher exact test, as appropriate. The Student $t$ test and the Mann-Whitney $U$ test were used for comparisons of continuous variables as appropriate. Significance was set as $P<.05$. Propensity score matching was performed to compare the bare group and the scaffolding group. SPSS Statistics, Version 22.0 (IBM) was used for calculations. Matching was performed using the MatchIt package in R statistical and computing software, Version 3.5.0 (http://www.r-project.org/). Variables with significant difference between groups were used as matching covariates. Groups were matched in a 1:2 ratio, with the nearest calculated propensity logit, with a caliper width of $\leq 0.20$ of the SD of the propensity score logit. Subsequently, aneurysm occlusion rates were compared using the $\chi^{2}$ test or Fisher exact test as appropriate.

\section{RESULTS}

A total of 105 patients with 127 aneurysms treated using the Silk device were included. Thirty-four patients were male, and 71 were female; the mean age of the cohort was 51.2 [SD, 11.2] years (range, 14-78 years). Eighty-four patients with 105 aneurysms were treated with a Silk device only (BG group), and 21 patients with 22 aneurysms were in the SG. The mean age was similar between BG (50.8 [SD, 10.9] years) and SG (54.5 [SD, 12.4] years) $(P=.8)$. There was also no difference between the 2 groups with 
Table 1: Characteristics of the patients and aneurysms before and after propensity score matching

\begin{tabular}{|c|c|c|c|c|c|c|}
\hline & \multicolumn{3}{|c|}{ Before Propensity Score Matching } & \multicolumn{3}{|c|}{ After Propensity Score Matching } \\
\hline & $\begin{array}{l}\text { Bare FD Group } \\
\quad(n=105)\end{array}$ & $\begin{array}{l}\text { Scaffolding Group } \\
(n=22)\end{array}$ & $\begin{array}{c}P \\
\text { Value }\end{array}$ & $\begin{array}{l}\text { Bare FD Group } \\
\quad(n=24)\end{array}$ & $\begin{array}{l}\text { Scaffolding Group } \\
\qquad(n=15)\end{array}$ & $\begin{array}{c}P \\
\text { Value }\end{array}$ \\
\hline $\operatorname{Sex}^{a}$ (female) & $77(73.3)$ & $12(54.5)$ & .08 & $18(75.0)$ & $7(46.6)$ & .072 \\
\hline Age $(y r)^{b}$ & $51(14-78)$ & $54.5(31-74)$ & .54 & $50(32-76)$ & $53(35.0-74.0)$ & .777 \\
\hline Location ${ }^{\mathrm{a}}$ (anterior) & $96(91.4)$ & $20(90.9)$ & $>.99$ & $19(79.1)$ & $13(86.6)$ & .685 \\
\hline Location $^{\mathrm{a}}$ (proximal) & $16(15.2)$ & $6(27.3)$ & .21 & $6(25)$ & $4(26.7)$ & $>.99$ \\
\hline Morphology ${ }^{a}$ (saccular) & $99(94.3)$ & $13(59.1)$ & $<.001^{\mathrm{C}}$ & $20(83.3)$ & $11(73.3)$ & .685 \\
\hline Size $^{b}$ & $7(4.5)$ & $13.1(10.7)$ & $<.001^{\mathrm{C}}$ & $9.8(5.9)$ & $9.9(5.1)$ & .939 \\
\hline Recurrence $^{\mathrm{a}}$ (yes) & $9(8.4)$ & $3(13.6)$ & .43 & $1(4.1)$ & $2(13.3)$ & .547 \\
\hline
\end{tabular}

Note:-Values are mean (range) for quantitative variables or numbers and percentages for qualitative variables.

${ }^{a}$ Count (percentage).

${ }^{\mathrm{b}}$ Mean (SD).

'Statistically significance.

Table 2: Follow-up imaging results before and after propensity score matching ${ }^{\mathrm{a}}$

\begin{tabular}{|c|c|c|c|c|c|c|}
\hline & \multicolumn{2}{|c|}{ All Cohorts } & \multirow[b]{2}{*}{$P$ Value } & \multicolumn{2}{|c|}{ After PSM } & \multirow[b]{2}{*}{$P$ Value } \\
\hline & Silk Alone $(n=105)$ & SG $(n=22)$ & & Silk Alone $(n=24)$ & SG $(n=15)$ & \\
\hline \multicolumn{7}{|c|}{0 - to 3-month follow-up } \\
\hline Occlusion $^{\mathrm{b}}$ & $36(60.0)$ & $5(33.3)$ & .063 & 7 (41.2) & $3(33.3)$ & $>.99$ \\
\hline Residual filling ${ }^{\mathrm{b}}$ & $24(40.0)$ & $10(66.6)$ & & $10(58.8)$ & $6(66.6)$ & \\
\hline \multicolumn{7}{|c|}{ 3- to 6-month follow-up } \\
\hline Occlusion ${ }^{\mathrm{b}}$ & $50(67.5)$ & $13(72.2)$ & .703 & $10(55.5)$ & $9(75.0)$ & .440 \\
\hline Residual filling ${ }^{\mathrm{b}}$ & $24(32.4)$ & $5(27.7)$ & & $8(44.5)$ & $3(25.0)$ & \\
\hline \multicolumn{7}{|c|}{ 7- to 12-month follow-up } \\
\hline Occlusion ${ }^{\mathrm{b}}$ & $61(75.3)$ & $14(93.3)$ & .178 & $13(65.0)$ & $9(90.0)$ & .210 \\
\hline Residual filling ${ }^{\mathrm{b}}$ & $20(24.6)$ & $1(6.6)$ & & $7(35.0)$ & $1(10.0)$ & \\
\hline \multicolumn{7}{|c|}{$>12$-month follow-up } \\
\hline Occlusion $^{\mathrm{b}}$ & 75 (88.2) & 15 (93.7) & $>.99$ & $14(73.6)$ & $11(91.6)$ & .363 \\
\hline Residual filling $^{\mathrm{b}}$ & 10 (11.7) & $1(6.2)$ & & $5(26.4)$ & $1(8.4)$ & \\
\hline
\end{tabular}

Note:-PSM indicates propensity score matching.

${ }^{a}$ Data are presented as numbers and percentages.

${ }^{\mathrm{b}}$ Count (percentage)

respect to the proportion of female patients $(P=.95)$, aneurysm location (anterior-versus-posterior circulation, $P>.99$, or proximal versus distal to the circle of Willis, $P=.21$ ), and the proportion of treatments performed for recurrent aneurysms $(P=.43)$. However, the aneurysms in the scaffolding group were significantly larger than those in the bare FD group (13.1 [SD, 10.7] $\mathrm{mm}$ versus 7 [SD, 4.5] $\mathrm{mm}, P<.001$ ), and the proportion of fusiform/dissecting aneurysms was higher in the scaffolding group $(40.9 \%$ versus $5.7 \%, P<.001)$. The level of platelet inhibition as per the VerifyNow test was similar between groups (mean, $83.0 \%$ [SD, 16.8\%] in the BG versus $86.1 \%$ [SD, $17.3 \%$ ] in the $\mathrm{SG}, P=.66$ ). Eleven aneurysms in the bare FD group and 2 aneurysms in the scaffolding group had intrasaccular embolization in addition to flow diversion, and both groups were similar with regard to the rate of adjunctive coiling $(P=.84)$.

Baseline characteristics of the patients are summarized in Table 1. In the scaffolding group, a LEO stent and a Silk device were combined in 19 patients; in 1 patient with a straight parent artery, an Enterprise stent and a Silk device were used; and in 1 patient, a LEO baby stent and a Silk Vista baby device were used. There was no significant difference in aneurysm occlusion rates at any followup period between treatment arms (Table 2). No imaging followup was available for $12(9.4 \%)$ aneurysms. Considering patients with at least 1 follow-up, 99 (86.0\%) aneurysms were occluded at last available imaging ( 80 of 94 [85.1\%] in the BG and 19 of 21 [90.4\%] in the SG, $P=.732)$.

Due to differences in aneurysm characteristics between the 2 groups, we performed propensity score matching to eliminate an inherent selection bias. After performing a 2:1 match based on aneurysm size, and morphology, 24 and 15 patients remained in the bare FD and scaffolding groups, respectively (Table 1). As expected, there was no difference between groups with respect to age; proportion of female patients; or aneurysm size, location, morphology, or history of recurrence (Table 1). Also, there were no significant differences between groups in terms of platelet inhibition levels $(77.6 \%$ [SD $19.6 \%$ ] in the BG versus $86.4 \%$ [SD, $18.5 \%$ ] in the SG, $P=.207$ ). There was no significant difference in the aneurysm occlusion rates between the groups in any of the 4 follow-up periods. More specifically, aneurysm occlusion rates were $41.2 \%$ versus $33.3 \%(P>.99)$ at $1-3$ months, $55.5 \%$ versus $75.0 \%(P=.44)$ at $3-6$ months, $65.0 \%$ versus $90.0 \%(P=.210)$ at $7-12$ months, and $73.6 \%$ versus $91.6 \%(P=.363)$ beyond 1 year for the bare FD and scaffolding groups, respectively (Table 2).

There was no difference in the rate of adverse events between groups (2/24 [8.3\%] versus $1 / 15$ [6.6\%], $P>.99)$. There were no technical events that resulted in an alteration of the treatment plan in any of the matched patients. In the bare FD group, 1 patient had a delayed stent occlusion leading to a major stroke; on follow-up, the patient had died due to the consequences of the 
stroke. Another patient was noted to have asymptomatic stent occlusion on the 6-month angiogram. In 1 patient in the scaffolding group, a small intraparenchymal hematoma was observed during the follow-up period, and the patient recovered without any permanent sequelae. No adverse events with neurologic implications developed in the remaining patients. Examples of aneurysms in both the bare FD and scaffolding groups are provided as Online Supplemental Data.

In the subgroup of patients with scaffolding stents who were not matched with propensity score matching, there were 7 patients. The average size of the aneurysms in this group was $20 \mathrm{~mm}$, and 2 were saccular. They were located at the supraclinoid internal carotid artery (2 patients), cavernous internal carotid artery (2 patients), middle cerebral artery (2 patients), and anterior communicating artery (1 patient). All were treated by a combination of LEO stents and Silk Plus flow diverters. There was no perioperative permanent morbidity or mortality in this group. One patient in this group died secondary to a contralateral cerebral parenchymal hematoma. The total occlusion rate was $33.3 \%$ at $1-3$ months. This had increased to $60 \%$ as of 6 months. At 12 months and beyond, it was $100 \%$.

\section{DISCUSSION}

Flow diversion has become a standard endovascular treatment method, especially for large or wide-neck aneurysms, which are difficult to treat with conventional treatment methods. Among the numerous flow-diverting devices, the Silk Plus flow diverter stands out with its navigability and smaller delivery catheter size. It has been used in the treatment of intracranial aneurysms with a high technical success rate, ${ }^{2,3,5}$ that is, complete aneurysm occlusion rates of approximately $70 \%-87 \%$ at 6 months to 1 year. ${ }^{2,5,7}$ However, the Silk Plus flow diverter is also associated with some technical difficulties regarding the treatment of complex aneurysms. ${ }^{8}$ To overcome these issues, the LEO stent has been used as a scaffolding device, compensating for the low radial force of the Silk Plus flow diverter, ${ }^{3}$ to prevent the prolapse of the device into the aneurysm ${ }^{8}$ or to prevent delayed-configurational device changes due to device oversizing. ${ }^{9}$ However, to date, a comparison of the safety and efficacy of this scaffolding technique over bare flow diversion has not been performed. In fact, placement of a flow diverter within a stent has been regarded as controversial or even contraindicated by many authors. ${ }^{6,10}$

On the other hand, our results indicate that scaffolding is not necessarily associated with a higher procedural risk nor is it related to a worse clinical or angiographic result during followup. We did not use the technique routinely, only when actual or possible problems related to device deployment or sizing existed. Consequently, it was used to treat large/giant aneurysms more often. However, once we performed propensity matching to eliminate confounding variables including aneurysm size, we observed again that neither the angiographic nor the clinical results were adversely affected by scaffolding. Because this technique was used as a bailout for bare FD placement, we can conclude that scaffolding increases technical success without significantly altering clinical or angiographic results. An analogy of our approach can be made with bailout stent placement used for balloon-assisted coiling in this regard.
Some possible explanations can account for the safety and efficacy of scaffolding stents in cases in which this technique needs to be used. They prevent foreshortening and prolapse of the flowdiverter stent into the aneurysm, which can be seen in up to $9.3 \%$ of large and giant aneurysms. ${ }^{11}$ In addition to the procedural hassles these 2 phenomena may create, they may also lead to fish mouthing or delayed endothelization in the longer term. ${ }^{9,12,13}$ Deployment of a device with a length of at least 3 times the length of the aneurysm neck is advised to reduce prolapse risk, ${ }^{7}$ which may be practically impossible in cases of long-segment fusiform aneurysms or may be associated with a higher rate of parent artery occlusion after flow diversion with the Silk device. ${ }^{14}$ In our cohort, we did not observe any of these adverse events.

Another advantage brought about by scaffolding is to avoid telescoping flow-diverter placement. In large/giant aneurysms, placement of up to 19 devices in a single aneurysm in up to 5 treatment sessions has been described. ${ }^{15}$ Scaffolding obviates the need for telescoping FDs and hence lowers the risk of perforator compromise associated with telescoping flow diverters. ${ }^{16}$ It also decreases the cost compared with telescoping FDs.

There is a strong preconception in the literature about preexisting stents being associated with failure of the flow diversion. This blanket statement was initially suggested by Nelson et $\mathrm{al}^{17}$ in the Pipeline Embolization Device for Uncoilable or Failed Aneurysms (PUFS) trial ${ }^{12}$ but has not been proved. It was cited and suggested by other authors as well. ${ }^{10,18-20}$ One critical point about this concept is that it relates exclusively to FDs deployed inside laser-cut stents. ${ }^{17-20}$ Incomplete opening or ovalization with laser-cut stents is well-known. ${ }^{8}$ Placement of a flow diverter inside a stent with pre-existing malapposition may be the reason for an endoleak at the landing zone and subsequent flow-diverter failure. ${ }^{10}$ Scaffolding performed by a properly deployed braided stent is unlikely to have malapposition. Indeed, the Flow-Redirection Endoluminal Device (FRED flow diverter; MicroVention) is a type of scaffolding stent/flow-diverter combination. With the exception of a single Enterprise stent deployed in a straight arterial segment, all of the stents in our scaffolding group were LEO stents, which have been shown, in vitro, to have good conformability to the vessel wall due to a reduced tendency to buckle or kink as opposed to laser-cut stents. ${ }^{21}$ We suggest that stents that are well-apposed to the arterial wall do not inevitably lead to failure of further endovascular treatment with FDs. Braided stents may be better suited for this application.

There are alternatives to the scaffolding method as well. Some authors advocate partial coiling of an aneurysm in cases prone to intra-aneurysmal device prolapse to "hold" the flow diverter in the parent artery, ${ }^{22}$ but this bailout technique may also be associated with complications. ${ }^{23}$ Dense coiling may lead to occlusion of the flow diverter due to increased thrombogenicity. ${ }^{24}$ In addition, the presence of coils within the sac makes noninvasive follow-up with CTA (and to some extent, with MRA) less reliable.

We chose the Silk device for the comparative analysis of scaffolding. This device is a nitinol-based device that, as its name implies, is very soft, navigable, and pliable. However, these benefits come at the expense of a propensity to foreshorten and, at times, a delay in configurational change. ${ }^{9,12}$ Hence, scaffolding stent placement has been used in a certain proportion of 
patients treated by authors who prefer to use this device. $^{1,3,5,25}$ The availability of matching sizes of the braided LEO stents for each Silk device size that can be deployed through the same delivery microcatheter also renders this technique attractive and relatively easy to perform. In our practice, most scaffolding stent placements accompanied the placement of the Silk device for these reasons. To eliminate the flow-diverter architecture and mechanics as confounding variables, we included in our study only aneurysms treated with the Silk device.

When placement of a scaffolding stent was planned, we followed several technical principles. To achieve a good wall apposition of the stent, we used only braided stents at the carotid siphon because closed-cell stents do not appose well in the siphon. We made sure that the stent was long enough to cover about $10 \mathrm{~mm}$ of the parent artery on both sides of the aneurysm to minimize the risk of stent migration into the aneurysm as we navigated the microcatheter through the deployed stent. We kept the tip of the delivery wire distal to the aneurysm to avoid recrossing of a freshly deployed stent with a guidewire, which may result in an inadvertent exit of the guidewire/delivery catheter from the stent lumen through the stent struts and then re-entry into the stent lumen at a distal point. Then, flow-diverter expansion will not be possible at the re-entry point, and if the scaffolding stent is an open-cell stent, the partially opened flow diverter may get entangled with it. Thus, we avoided open-cell stents and also stents without a distal delivery wire.

For stent delivery, we preferred microcatheters with angled tips (such as a multipurpose curve or $90^{\circ}$ angle) because the tip of the delivery microcatheter frequently got caught by a stent strut during the crossing of the deployed stent. If this situation occurred, we refrained from pushing the microcatheter further but, instead, pulled the microcatheter back slightly, torqued it gently, pulled or pushed the wire slightly, and then advanced the microcatheter, trying to avoid hitting the spot on the stent at which the microcatheter got caught. Finally, as long as optimal expansion and wall apposition of the scaffolding stent was clearly documented and the flow diverter was deployed only at the aneurysmal segment, we did not intentionally try to cover the entire length of the stent by the flow diverter.

The main limitations of this study include its retrospective nature and the relatively small sample size that remained after matching of the heterogeneous groups by propensity score analysis. Due to the retrospective nature, the imaging follow-up at 13 months, 3-6 months, 7-12 months, and beyond 1 year was not consistent for every case. In addition, success rates may be lower with other stent types because matching stent/flow-diverter pairs that are deliverable through the same microcatheter are not available for all devices on the market. This issue may mandate the use of an exchange maneuver once the stent is deployed, ${ }^{26}$ increasing the risk and complexity of flow diversion with some devices. However, as the first study on the comparative efficacy and safety of scaffolding stents, our study highlights the critical points in scaffolding stent placement. It also justifies the need for larger-scale prospective studies or verification of these results in larger cohorts.

\section{CONCLUSIONS}

Scaffolding stent placement to help flow diversion of intracranial aneurysms has been criticised by many authors. This may be the result of earlier publications that disparaged previously placed stents as a cause of flow-diversion failure. We propose that when flow diversion with a single FD is risky or impossible, scaffolding can be used with a good rate of success and without a significant increase in complications.

\section{REFERENCES}

1. Briganti F, Napoli M, Leone G, et al. Treatment of intracranial aneurysms by flow diverter devices: long-term results from a single center. Eur J Radiol 2014;83:1683-90 CrossRef Medline

2. Pumar JM, Banguero A, Cuellar H, et al. Treatment of intracranial aneurysms with the Silk embolization device in a multicenter study: a retrospective data analysis. Neurosurgery 2017;81:595-601 CrossRef Medline

3. Strauss I, Maimon S. Silk flow diverter in the treatment of complex intracranial aneurysms: a single-center experience with 60 patients. Acta Neurochir (Wien) 2016;158:247-54 CrossRef Medline

4. Lubicz B, Collignon L, Raphaeli G, et al. Flow-diverter stent for the endovascular treatment of intracranial aneurysms: a prospective study in 29 patients with 34 aneurysms. Stroke 2010;41:2247-53 CrossRef Medline

5. Piano M, Valvassori L, Quilici L, et al. Midterm and long-term followup of cerebral aneurysms treated with flow diverter devices: a singlecenter experience. J Neurosurg 2013;118:408-16 CrossRef Medline

6. Park MS, Taussky P, Albuquerque FC, et al. Off-label use. In: Rivet DJ II, Reavey-Cantwell J, Moran CJ, eds. Flow Diversion of Cerebral Aneurysms. Thieme 2017; 40-63

7. Maimon S, Gonen L, Nossek E, et al. Treatment of intra-cranial aneurysms with the Silk flow diverter: $\mathbf{2}$ years' experience with $\mathbf{2 8}$ patients at a single center. Acta Neurochir (Wien) 2012;154:979-87 CrossRef Medline

8. Gurkas E, Kaya T, Daglioglu E, et al. Silk device for the treatment of intracranial aneurysms, Part 1: peri-procedural results, technical aspects and learning curve. Turk Neurosurg 2016;26:525-32 CrossRef Medline

9. Peker A, Akgul E, Daglioglu E, et al. Tapered flow diverters in the treatment of intracranial aneurysms. Turk Neurosurg 2017;27:86366 CrossRef Medline

10. Shapiro M, Becske T, Nelson PK. Learning from failure: persistence of aneurysms following Pipeline embolization. J Neurosurg 2017;126:578-85 CrossRef Medline

11. Srinivasan VM, Carlson AP, Mokin M, et al. Prolapse of the Pipeline Embolization Device in aneurysms: incidence, management, and outcomes. Neurosurg Focus 2017;42:E16 CrossRef Medline

12. Cohen JE, Gomori JM, Moscovici S, et al. Delayed complications after flow-diverter stenting: reactive in-stent stenosis and creeping stents. J Clin Neurosci 2014;21:1116-22 CrossRef Medline

13. Shapiro M, Raz E, Becske T, et al. Variable porosity of the Pipeline embolization device in straight and curved vessels: a guide for optimal deployment strategy. AJNR Am J Neuroradiol 2014;35:72733 CrossRef Medline

14. Macdonald IR, Shankar JJS. Delayed parent artery occlusions following use of Silk flow diverters for treatment of intracranial aneurysms. J Neurointerv Surg 2019;11:690-93 CrossRef Medline

15. Bhogal P, Pérez MA, Ganslandt O, et al. Treatment of posterior circulation non-saccular aneurysms with flow diverters: a single-center experience and review of 56 patients. J Neurointerv Surg 2017;9:47181 CrossRef Medline

16. Chalouhi N, Tjoumakaris S, Phillips JL, et al. A single Pipeline Embolization Device is sufficient for treatment of intracranial aneurysms. AJNR Am J Neuroradiol 2014;35:1562-66 CrossRef Medline 
17. Nelson PK, Lylyk P, Szikora I, et al. The Pipeline Embolization Device for the Intracranial Treatment of Aneurysms Trial. AJNR Am J Neuroradiol 2011;32:34-40 CrossRef Medline

18. Daou B, Starke RM, Chalouhi N, et al. Pipeline Embolization Device in the treatment of recurrent previously stented cerebral aneurysms. AJNR Am J Neuroradiol 2016;37:849-55 CrossRef Medline

19. Heiferman DM, Billingsley JT, Kasliwal MK, et al. Use of flow-diverting stents as salvage treatment following failed stent-assisted embolization of intracranial aneurysms. J Neurointerv Surg 2016;8:692-95 CrossRef Medline

20. Bender MT, Vo CD, Jiang B, et al. Pipeline embolization for salvage treatment of previously stented residual and recurrent cerebral aneurysms. Interv Neurol 2018;7:359-69 CrossRef Medline

21. Ebrahimi N, Claus B, Lee CY, et al. Stent conformity in curved vascular models with simulated aneurysm necks using flat-panel CT: an in vitro study. AJNR Am J Neuroradiol 2007;28:823-29 Medline
22. Ferrigno AS, Caro-Osorio E, Martinez HR, et al. Coiling as a rescue strategy for flow diverter prolapse into a giant intracranial aneurysm. World Neurosurg 2020;133:392-97 CrossRef Medline

23. Trivelato FP, Wajnberg E, Rezende MT, et al. Safety and effectiveness of the Pipeline Flex embolization device with shield technology for the treatment of intracranial aneurysms: midterm results from a multicenter study. Neurosurgery 2020;87:104-11 CrossRef Medline

24. Siddiqui AH, Kan P, Abla AA, et al. Complications after treatment with Pipeline embolization for giant distal intracranial aneurysms with or without coil embolization. Neurosurgery 2012;71:E509-13; discussion 13 CrossRef Medline

25. de Andrade GC, Alves HP, Clímaco V, et al. Two-stage reconstructive overlapping stent LEO+ and Silk for treatment of intracranial circumferential fusiform aneurysms in the posterior circulation. Interv Neuroradiol 2016;22:516-23 CrossRef Medline

26. Ocal O, Arat A. The exchange-free technique: a novel technique for enhancing Surpass flow diverter placement. Asian J Neurosurg 2020;15:620-26 CrossRef Medline 